International Journal of Engineering \& Technology, $7(2.4)(2018) 56-58$
International Journal of Engineering \& Technology
WPC
Website: www.sciencepubco.com/index.php/IJET
Research Paper

\title{
Development of smart farming - a detailed study
}

\author{
C. M. Chidambaranathan ${ }^{1 *}$, S.S. Handa ${ }^{2}$, M.V. Ramanamurthy ${ }^{3}$ \\ ${ }^{1,2}$ Dept of CSE, SRM University, Delhi-NCR, Sonepat, India \\ ${ }^{3}$ Dept of CSE, Osmania University, Hyderbad,India \\ *Corresponding author E-mail: chidambaranathancm@srmuniversity.ac.in
}

\begin{abstract}
Agriculture is the backbone of every country. The country's development is not due to Industrial development alone, but also due to the agriculture development. The Country like India depends upon the 70 percent of the agriculture. In the recent year's agriculture productions got down due to the natural disasters like flood, famine and etc., hence the country should implement the latest technology to overcome the problems in agriculture. Recently Internet of Things (IoT) is the latest technology used in all the fields. By 2020, IoT would be effectively implemented in every field. In agriculture, Smart Farming which is involves connecting sensors and internet to collect various parameters like temperature, humidity, moisture and etc., and share with the farmers periodically. It is the latest technology which improves the agriculture as well as country economy. In this paper we will discuss the various methods of smart farming.
\end{abstract}

Keywords: WSN-Wireless Sensor Network, IoT-Internet of Things, Gateway Access, ZigBee module.

\section{Introduction}

Smart Farming is the latest development; the technology allows farmers to improve the efficiency and value of all managed resources. The technology provides the detailed and adoptive innovative practices to deliver sustainable agriculture. This is where exactly smart farming plays a major role. Devices like Sensors, actuators and Global Positioning System (GPS) devices are available for the effective agriculture; engineering technologies always provides the farmers with innovative technologies for protecting the valuable resources. With proper planning, implementation, and execution of technology will yield very good result in agriculture. Smart farming is perfectly suitable for the unique Integrated Farming concept. In daily life farmers are facing different problems like natural disaster; unexpected fire, insect attacks etc., and this paper discuss various systems which is helpful in monitoring the environment and controlling the sensor nodes to provide proper information to farmers. Smart farming aims to make agriculture completely in automation for the high yield. The highlighting features of this survey paper includes the various technologies used in smart farming were discussed. Smart irrigation and smart warehouse management which controls; temperature, moisture, humidity in the sensor nodes. Managing of all these operations will be carried out by wireless or internet connected devices and the operations will be performed by various sensors, actuators, and Wi-Fi or ZigBee modules. This paper discusses the different techniques used in smart farming.

\section{Overview of smart farming and wireless sensor network}

Smart farming is the ability to handle variations in agriculture productivity within a field and maximize economy, reduce residues and minimize impact of the environment impacts using automated data collection, documentation and utilization of such information for strategic farm management decisions through sensors and actuators. Several technologies were used in the Smart farming such as Remote Sensing (RS), Geographic Information System (GIS) and Global Positioning System (GPS). The Wireless sensor network (WSN) is composed of a large number of sensor nodes which collects data and transmitted to the base station. These nodes are densely implemented in the agricultural field Sensor nodes coordinate among themselves based on the various protocols and delivers high-quality of information about the field environment. Each of these distributed sensor nodes has the capacity to collect and route data either to other sensors or back to the base stations or servers .Base station connects all the wireless sensor network via internet where the farmers could receive real time data.

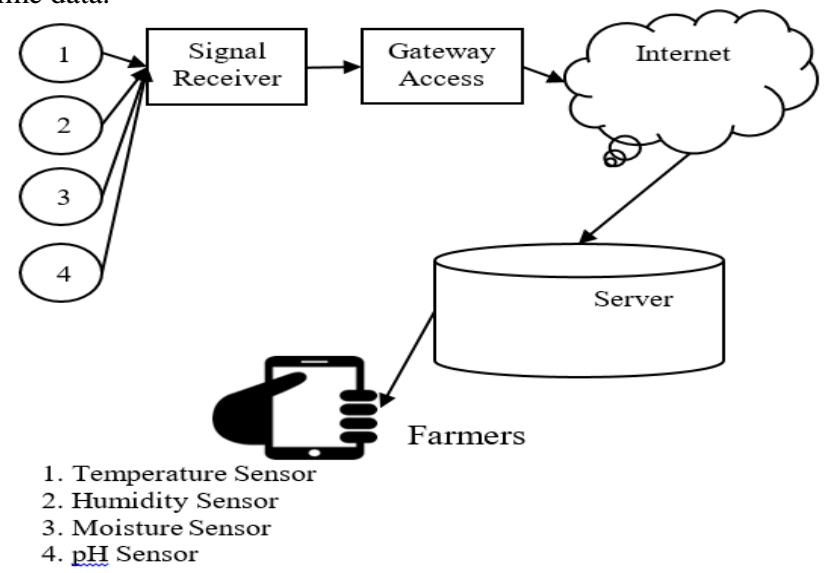

Fig 1: System Architecture of Smart Farming using Wireless Sensor Network

The above diagram shows the data collection from sensor nodes and transmitted to server through signal receiver and those collected information is shared with farmers.

- Sensor nodes - Sensor nodes are mounted in the different places of the field. The sensors collect temperature, moisture, humidity, $\mathrm{pH}$ etc. 
- Signal Receiver- Signal receives the data from sensors and route to the Gateway.

- Gateway - A Gateway enables communication between sensor nodes and host applications.

\section{Related works}

Laith Ali Abdul-Rahaim proposed the new programming technology which allows the network to adjust the data values itself. It also adjusts the nodes values, and nodes count. Radio Frequency setup has been implemented to send and receive data through sensors. Though the system is intra network, the other security system like video monitoring system (IP) and cameras connected with a server for monitoring the environment. The proposed system contains number of precision sensors implemented in network nodes to measure the actual values of environmental parameters like (temperature, humidity, and water irrigation level, $\mathrm{PH}$ content in the water) and transmit them to the base station "Gateway" in the field. The videos sent by cameras are very helpful to monitor the environment. Remote monitoring will reduce the labor and other expenses. Simultaneously the system must handle the errors coming from the sensors. The entire network can be implemented using Graphical representation format called Lab View program [1].

Syed Ijlal Ali Shah developed a multi-tiered hybrid network architecture which consists of multiple single-hop sensors, this system is a wireless sensor networks (WSNs)-based network used for the efficient water management application. The proposed architecture can handle the fault-tolerance and modularity. The system also monitors the network during natural disasters. The network can handle the situation if it completely fails but part of the network will function. The system contains Sensing device which is responsible for collecting information. The collected information is stored in the Collection Agent. The relay device is used to transmit data to the host (Data Depot) [2].

Juan A. Lopez developed the architecture for monitoring crops which are wide spread up to several distance. Juan developed the B-MAC protocol (MAC (Berkeley Medium Access Control) to avoid the power autonomy of sensor nodes. To overcome this issue the network has been implemented with the series of sensor nodes interconnected to the server. Also the system has been implemented in star topology to resolve the power autonomy issue. Data frame holds the information segment at the MAC layer. It reduces the usage of wireless devices. Finally, the simulation process ensures the gateway in the network will transmit all the frames with spaced intervals to overcome the collisions. The device comes with the photovoltaic solar panel to reduce the energy consumption [3].

Santhosh Simon defines the implementation of ZigBee modules such as security and application layer provides the compatibility between different devices. ZigBee implementation minimizes the power consumption, reduced cost and network infra structure. The sensors implementation in ZigBee technique can handle the severe environment condition and deserted locations. Mainly this system has been implemented in paddy field to monitor the water level. The sensors nodes are fixed on the sensor columns and the sensors are used to check the water level. The sensor nodes convert analog value into digital value. After converting into digital the data is transmitted into server or host. So the future work will be in the power consumption [4].

Orazio Mirabella discussed various problems related to green houses. The problem which has been identified in the green house is data transfer. The data collected from sensors installed in green house will be transfer to the control unit located in Main room or server room. Instead of using wired communications Orazio used Smart Distributed System (SDS) which permits the implementing on Controller Area Network (CAN) protocol which is used for the automation system. The reason for chose SDS system is very convenient to implement on various resource devices. It is well adaptable for monitoring green houses [5].
P.R.S. Rao et al. have done survey on the various problems where farmer faced during the data collection from the sensor nodes. Rao implemented the new technology called COMMON-Sense Net System which is useful to monitor the rain fed agriculture. The system minimizes the water resources based on the information received from the sensor. Rao examined the results over two year period with different characteristics of soil and air content. Moreover the system architecture was made by Cluster-based Hybrid model for the data transmission through GPRS technology [6].

Manikandan et al. implemented the Smart Irrigation Application system which combines with the help of RF $433 \mathrm{MHz}$ modules and micro controller which is used to convert the collected data into desired results. To collect the moisture and temperature data from the corresponding sensors installed in the field. So the control unit controls the flow of water and soil content. This system reduces the water wastage. Present work develops low cost sensors for monitoring the system. Whenever the soil temperature increases or moisture varies the Automatic Drip Irrigation controls the system and maintains the water level [7].

Davide Di Palma et al. discussed various problems in wired network infrastructure and those problems can be resolved by Wireless Sensor Network for the effective agriculture. Instead of controlling different sensors a compliant sensor board has been adopted, it provides the platform for all sensors in the same mode. It is very convenient for sensing different environmental parameters. To optimize the larger radio coverage and increase the power management the system has been implemented with several antennas and proper circuit along with the power amplifier for enhance the transmitting power according to IEEE standards. To avoid the node failure due to long time running and idle for longer period MAC protocol is used. To provide the exact result and calculate the redundancy, High sensor density was implemented in the field [8]

Santhosh Simon states the advantages of Wireless Sensor Network efficiently used in the precision agriculture. The advantages of precision agriculture are distributed real time data collection and monitoring the environmental parameters. Periodic checking and controlling of those parameters like (humidity, temperature, and soil content) are necessity for the paddy growth. Santhosh implemented the ZigBee network, because the setup cost is low and less power consumption. ZigBee module was implemented on IEEE 802.15.4. ZigBee cluster head reads the value from the sensor nodes. During critical condition the cluster head goes to alert state and transmits the data packets directly to the sink node. The sink node instructs all the sensors to sniff state which means until the sensors goes to normal state the sink node keep on instructing to all sensors regarding the critical situation like water level increasing or flood etc. Once the cluster head receives the proper value from the sensor that is normal condition the sink node instructs all the sensors to go sleep state. Usually sensors possess two states: sniff and sleep state. Once the entire field comes under the normal condition the entire network will remains in sleep state [9].

Mrs.Tejal K. Joshi et al. implemented an environment monitoring system for effective agriculture based on wireless sensor network. The system collects humidity, illumination voltage, moistures and various parameters and transmits to server using GPRS technology. In this technology TelosB sensors were used to collect the data MSP 430F1611 and CC2420 chips were used in the sensor nodes. This chip maintains the consistency and increase the battery life of sensors [10].

Pratibha Gangurde et al. implemented the energy consumption and compactable network in Precision agriculture. Usually the sensor nodes communicate via shortest paths for the data sharing. Also the packets are shorter. The energy of wireless sensor network would be saved by scheduling of the nodes, altering transmission range of sensor nodes and proper routing of data collection during eavesdropping and regret the unwanted data sharing. Sensors transmit the data to the sink node or server if critical condition happens. This would be taken in single or Multihop topology based on the location of the sensors. So the new methodology eradicates the individual requirements of different characteristics 
and application of crops a collection of common tasks which are used to reduce the power consumption and enhance the agricultural productivity. Using this methodology the new way of optimal sensor network is implemented for reducing energy consumption and minimal cost [11].

Luis Ruiz-Garcia implemented the CAN technology which enhances the transport monitoring and promises the affinity of the system. The CAN technology built with various sensors and actuators. Periodically the data has been monitored and warn the nodes during environmental impacts. Nowadays interconnecting the diverse systems and various interfaces is the major problem in the wireless sensor network. The new technology "Smart Container" holds the different sensors in the same system. Fixed-Priority Scheduling (FPS) is implemented in the CAN medium access control (MAC) layer using the bit-wise arbitration mechanism. CAN technology resolve the time collision problem. Also ZigBee protocol was used in this technology for the power consumption [12].

Luis Ruiz-Garcia et al. reviewed various wireless sensor technologies and wireless communications standards in the Agricultural and food sector. Luis Ruiz-Garcia et al. focuses on WSN (Wireless Sensor Networks) and RFID (Radio Frequency Identification), along with the ZigBee based Wireless Sensor Network with RFID which is partially active and passive. The system is integrated with sensor nodes and every sensor nodes communicate via LAN, WLAN, CAN, GSM etc. The systems are usually composed of a few sinks and large quantity of small sensors nodes. RFID has been mostly implemented in supply chain management, cold storage and food logistics [13].

$\underline{\text { Jose Polo }}$ et al. designed a new low cost Wireless Network for agricultural purpose. Multiple sensors are placed in different places and the mobile node which is responsible for data collection. Sometimes mobile nodes take long time for data collection and could not provide real time data. To cover the long distance with in a limited time Jose designed a new technology called Unmanned Aerial Vehicle (UAV). The vehicle travel along the field and collect the real time data like soil moisture, crops disease and etc [14]

Cheick Tidjane Kone et al. discussed the traffic control problem in WSNs using in precision agriculture. The monitoring and control of crops in smart farming sometimes requires a huge collection frequency of information like (temperature, moisture, humidity) due to the volatility of crops. Data gathering and transmission are carried out by Wireless Sensor Network. Due to shortage of sensor nodes it is mandatory to increase the sampling frequency for different crops. To overcome this problem, Kone proposed properly tuned IEEE 802.15.4 MAC parameters (macMinBe and macMaxCSMABackoffs) and sampling frequency of sensor nodes. It provides high spatial granularity of observations for precision agriculture or other WSN applications [15].

\section{Conclusion and future work}

Smart farming will be self-sustained once all the issues are sorted out by introducing efficient protocols and emerging technologies. This survey provides the various issues in Wireless Sensor Network during the data collection and transmission to the base station in particular, data loss and data packet delay are the major issues that has to be resolve. The monitoring of the nodes in different places is a difficult task, to overcome these issues, medium access protocol such as B-MAC (Berkeley Medium Access Control) has to be implemented to assure a high degree of reliability and efficiency. In some cases the sensor nodes response time gets delay and trade-off occurs. In order to provide periodic information without any data loss, work need to be carried out using effective protocols to alleviate the tradeoff between energy efficiency and time delay between nodes

\section{References}

[1] Laith Ali Abdul-Rahaim et al., "Remote Wireless Automation and Monitoring of Large Farm using wireless sensors networks and Internet", International Journal of Computer Science \& Engineering Technology (IJCSET) Vol. 6 No. 03 Mar 2015.

[2] Syed Ijlal Ali Shah, Marwan Fayed, "AQUA-NET: a flexible architectural framework for water management based on wireless sensor networks", IEEE, Electrical and Computer Engineering (CCECE), 2011.

[3] Juan A. Lopez et al. "Design and validation of a wireless sensor network architecture for precision horticulture applications", Springer, Precision Agric (2011) 12:280-295, 2010

[4] Santhosh Simon, K Paulose Jacob "Energy Optimized Secure Routing Protocol for Wireless Sensor Networks" International Journal of Engineering and Innovative Technology (IJEIT) Volume 3, Issue 4, October 2013.

[5] Orazio Mirabella, Michele Brischetto "A Hybrid Wired/Wireless Networking Infrastructure for Greenhouse Management", IEEE TRANSACTIONS ON INSTRUMENTATION AND MEASUREMENT, VOL. 60, NO. 2, FEBRUARY, 2011.

[6] J.Panchard, et al. ,"Wireless Sensor Networking for Rain-fed Farming Decision Support", Tech.Rep, ACM SIGCOMM Workshop on Networked Systems for Developing Regions, (NSDR),USA, Vol 1,0912.5506 [cs.NI], 2009.

[7] Manikandan, "Automatic Monitoring System for a Precision Agriculture Based On Wireless Sensor Networks", IJCSET , June 2016 | Vol 6, Issue 6, 208-211, 2016

[8] Davide Di Palma et al. , "Distributed Monitoring Systems for Agriculture based on Wireless Sensor Network Technology", International Journal on Advances in Networks and Services, Vol 3 - 1 \& 2, 2010.

[9] Santhosh Simon, "Sensor Networks for Paddy Field Crop Monitoring Application", International Journal of Modern Engineering Research (IJMER) Vol.2, Issue.4, July-Aug 2012 pp-20172020.

[10] Mrs.Tejal K. Joshi et al., "Wireless sensor network solution for precision agriculture", International Journal for Technological Research in Engineering Volume 3, Issue 4, December-2015.

[11] Pratibha Gangurde et al., "A Novel Approach for Precision Agriculture Using Wireless Sensor Network", International Journal of Computer Science and Mobile Computing, Vol.4 Issue.6, June- 2015, pg. 1158-1165.

[12] Luis Ruiz-Garcia et al., "CAN-based monitoring in refrigerated transports", ICC 2008.

[13] Luis Ruiz-Garcia et al., "A Review of Wireless Sensor Technologies and Applications in Agriculture and Food Industry: State of the Art and Current Trends", Sensors 2009, 9, 47284750.

[14] Jose Polo et al., "Design of a low-cost Wireless Sensor Network with UAV mobile node for agricultural applications", 2015.

[15] Cheick Tidjane Kone et al., "Performance Management of IEEE 802.15.4 wireless sensor network for precision agriculture", 10.1109/JSEN.2015.2442259, IEEE Sensors Journal. 